\title{
SOME ARITHMETIC MEANS CONNECTED WITH FOURIER SERIES*
}

\author{
BY \\ L. S. BOSANQUET
}

1. Introduction. It is known that if a series $\sum a_{n}$ is bounded $(C, \gamma), \gamma \geqq-1$, then it is either summable $(C, \gamma+\delta)$, for every $\delta>0$, or not summable $(A) . \dagger$ Further a necessary and sufficient condition for it to be bounded $(C, \gamma)$ is that it should be bounded $(A)$ and the sequence $n a_{n}$ bounded $(C, \gamma+1)$. $\ddagger$ Conditions have been obtained $\S$ under which a Fourier series $\sum A_{n} \cos n t$ or an allied series $\sum B_{n} \cos n t$ should be bounded $(C)$ for $t=0$ and, in the case of the allied series, conditions under which the sequence $n B_{n}$ should be bounded $(C)$. But the problem of the boundedness $(C)$ of the sequence $n A_{n}$ has apparently not been considered directly.\|

We suppose that $f(t)$ is integrable $L$, and periodic with period $2 \pi$. We write

$$
\begin{aligned}
& \phi(t)=\frac{1}{2}\{f(x+t)+f(x-t)\}, \\
& \psi(t)=\frac{1}{2}\{f(x+t)-f(x-t)\},
\end{aligned}
$$

and suppose that the Fourier series of $\phi(t)$ and $\psi(t)$ are respectively

$$
\sum_{n=0}^{\infty} A_{n} \cos n t \text { and } \sum_{n=1}^{\infty} B_{n} \sin n t .
$$

Then the Fourier series and allied series of $f(t)$ at the point $t=x$ are respectively

$$
\sum_{n=0}^{\infty} A_{n} \quad \text { and } \quad \sum_{n=1}^{\infty} B_{n} . \text { ๆ }
$$

We write, for $t>0$,

* Presented to the Society, September 13, 1935; received by the editors March 5, 1935.

$\dagger$ Proved by Littlewood, 17, for $\delta=1$ and $\gamma=$ integer, and completed by Andersen, 1. See also Hardy and Littlewood, 13a, Kogbetliantz, 15, p. 38. Numbers in heavy type refer to the list of references at the end of this paper.

$\ddagger$ Hardy and Littlewood, 13a, p. 283. Kogbetliantz, 15, p. 38.

$\S$ The first systematic results of this type were given by Hardy and Littlewood, 11, 12.

$\|$ Sufficient conditions for the existence of the Cesàro limits of $n A_{n}$ and $n B_{n}$ have been given by Young, 24, 25, and the limits of the arithmetic and logarithmic means of $n B_{n}$ have been considered by other writers. See Zygmund, 27, where references are given.

If In the usual notation $A_{0}=\frac{1}{2} a_{0}, A_{n}=a_{n} \cos n x+b_{n} \sin n x$ and $B_{n}=b_{n} \cos n x-a_{n} \sin n x, n>0$. 


$$
\begin{aligned}
\theta(t) & =\frac{2}{\pi} \int_{t}^{\infty} \frac{\psi(u)}{u} d u, \\
\Phi_{\alpha}(t) & =\frac{1}{\Gamma(\alpha)} \int_{0}^{t}(t-u)^{\alpha-1} \phi(u) d u, \quad \alpha>0, \\
\Phi_{0}(t) & =\phi(t), \\
\Phi_{\alpha}(t) & =\frac{d}{d t} \Phi_{\alpha+1}(t), \quad-1<\alpha<0, \\
\phi_{\alpha}(t) & =\Gamma(\alpha+1) t^{-\alpha} \Phi_{\alpha}(t), \quad \alpha>-1, \\
\phi_{-1}(t) & =\frac{d}{d t}\{t \phi(t)\},
\end{aligned}
$$

and we define $\Psi_{\alpha}(t), \psi_{\alpha}(t), \Theta_{\alpha}(t), \theta_{\alpha}(t)$ in a similar way. We call $\phi_{\alpha}(t)$ the mean value of order $\alpha$ of $\phi(t)$.

We also write $s_{n}^{\alpha}, \bar{s}_{n}^{\alpha}, \tau_{n}^{\alpha}, \bar{\tau}_{n}^{\alpha}$ for the Cesàro means of order $\alpha$ of $s_{n}=A_{0}+A_{1}+\cdots+A_{n},{ }^{*} \bar{s}_{n}=B_{1}+B_{2}+\cdots+B_{n}, \tau_{n}=n A_{n}$ and $-_{n}=n B_{n}$ respectively. Finally we write, for $\alpha \geqq 0$,

$$
R_{\alpha}(\omega)=\sum_{n<\omega}(\omega-n)^{\alpha} A_{n}, \quad \bar{R}_{\alpha}(\omega)=\sum_{n<\omega}(\omega-n)^{\alpha} B_{n},
$$

and

$$
r_{\alpha}(\omega)=\omega^{-\alpha} R_{\alpha}(\omega), \quad \bar{r}_{\alpha}(\omega)=\omega^{-\alpha} \bar{R}_{\alpha}(\omega) . \dagger
$$

It is known $\ddagger$ that if $\left|\phi_{\alpha}(t)\right|=O(1)(C, 1)$, i.e.,

$$
\frac{1}{t} \int_{0}^{t}\left|\phi_{\alpha}(u)\right| d u=O(1)
$$

in an interval $(0, \eta)$, then $s_{n}^{\beta}=O(1)$ for $\beta>\alpha$, where $\alpha \geqq 0$. The Cesàro summability and boundedness of a Fourier series for negative orders is not a "local" property of the function, since we usually only know that $A_{n}=o(1)$, but the above result remains true when $-1 \leqq \alpha<0$ if we take $\eta=\pi$,

* Thus

$$
s_{n}^{\alpha}=\frac{1}{A_{n}^{\alpha}} \sum_{\nu=0}^{n} A_{n \rightarrow \nu}^{\alpha-1} s_{\nu},
$$

where

$$
A_{n}^{\alpha}=\frac{\Gamma(\alpha+n+1)}{\Gamma(\alpha+1) \Gamma(n+1)} .
$$

$\dagger$ Thus $r_{\alpha}(\omega), \bar{r}_{\alpha}(\omega)$ are the Rieszian arithmetic means of order $\alpha$ of the Fourier series and allied series respectively.

‡ See Bosanquet, 6, where references to previously known special cases are given. 
provided $\Phi_{\alpha+1}(t)$ is absolutely continuous and $\Phi_{\alpha+1}(+0)=0$, in the case $\alpha>-1,{ }^{*}$ and $\phi(t)$ is absolutely continuous except at $t=0$ in the case $\alpha=-1$. Dr. A. C. Offord and I have recently shown that for the class of Fourier series for which $A_{n}=o\left(n^{\gamma}\right), \gamma>-1$, summability $(C, \gamma)$ at the point $t=x$ does in fact depend only on the local properties of the function, and there is an analogous result for boundedness $(C)$. It follows from our conditions that, if we restrict ourselves to series for which $A_{n}=O\left(n^{\beta}\right)$, the above result remains true, with the condition in its localized form, when $\alpha \geqq-1$. $\dagger$ Condition (1) may also be replaced by the more general form

$$
\frac{1}{t} \int_{0}^{t} u^{-\alpha}\left|d \Phi_{\alpha+1}(u)\right|=O(1) \ddagger
$$

when $\alpha>-1$, or

$$
\frac{1}{t} \int_{0}^{t}|d\{u \phi(u)\}|=O(1)
$$

when $\alpha=-1 . \S$ There are also "converse" results, $\|$ in which summability or boundedness of the Fourier series is given as an hypothesis. It follows in particular from these results that a necessary and sufficient condition that $s_{n}^{\beta}=O(1)$ for some $\beta$ is that (1) should hold for some $\alpha$, or, what is the same thing, that $\phi_{k}(t)=O(1)$ for some $k$.

The investigations of this paper arise out of certain identities which play an important role in the theory of Cesàro means. If $\alpha>0$, we have

$$
\alpha\left(s_{n}^{\alpha-1}-s_{n}^{\alpha}\right)=n\left(s_{n}^{\alpha}-s_{n}^{\alpha}-1\right)=\tau_{n}^{\alpha}, \prod
$$

and ** $^{* *}$

$$
\alpha\left\{\phi_{\alpha-1}(t)-\phi_{\alpha}(t)\right\}=t \phi_{\alpha}{ }^{\prime}(t)=\chi_{\alpha}(t)
$$

* In these circumstances $\Phi_{\alpha+1}(t)=\int_{0}^{t} \Phi \alpha(u) d u$. The last condition is necessary in order to exclude a function like $f(t)=|t-x|^{-\alpha-1},-1<\alpha<0$, whose Fourier series diverges for $t=x$. If $\phi(t)=|t|^{-\alpha-1}$, we have $\Phi_{\alpha+1}(t)=\Gamma(-\alpha)$, and hence $\phi_{\alpha}(t)=0$ for every $t>0$.

$\dagger$ See Bosanquet, 8, Bosanquet and Offord, 9.

$\ddagger$ An integral like (2) is to be interpreted in the first instance as $\lim _{\epsilon \rightarrow 0} \int_{\epsilon}^{t}$, where it is assumed that $\Phi_{\alpha+1}(u)$ is of bounded variation in every interval $(\epsilon, t)$. But when condition (2) is satisfied it may be shown that $\Phi_{\alpha+1}(u)$ is of bounded variation in $(0, t)$, and so the integral exists as an ordinary LebesgueStieltjes integral. See Bosanquet, 8.

$\S$ Condition (3) is Young's well known condition. Young, 26, stated that it was sufficient for boundedness $(C,-1+\delta)$, for $\delta>0$, and this was proved by Hardy and Littlewood, 13, in the case $\eta=\pi$.

\| See Theorem 6, p. 201, where further references are given.

T. Kogbetliantz, 15, and 16, pp. 23 and 30.

** For (5) and (6) see Bosanquet, 6 and 7. The analogy between these and (4) is obvious. 
where $\chi_{\alpha}(t)$ is the mean value of order $\alpha$ of $t \phi^{\prime}(t)$ whenever $t \phi(t)$ is an integral vanishing at $t=0$. We also have

$$
\alpha\left\{\theta_{\alpha-1}(t)-\theta_{\alpha}(t)\right\}=t \theta_{\alpha}^{\prime}(t) \equiv-\frac{2}{\pi} \psi_{\alpha}(t) .
$$

It will be shown here that, if

$$
\frac{1}{t} \int_{0}^{t}\left|\phi_{\alpha}(u)-\phi_{\alpha+1}(u)\right| d u=O(1)
$$

in an interval $(0, \eta)$, then

$$
s_{n}^{\beta}-s_{n}^{\beta+1}=O(1)
$$

for $\beta>\alpha$, i.e., $n A_{n}=O(1)(C, \beta+1)$. This is true with any $\eta, 0<\eta \leqq \pi$, when $\alpha \geqq 0$, and with $\eta=\pi$ when $\alpha \geqq-1$, provided $\phi_{\alpha+1}$ is absolutely continuous except at $t=0$. For the class of Fourier series for which $A_{n}=O\left(n^{\beta}\right)$ there is a corresponding "localization" problem, but that belongs to a rather different line of ideas and will not be discussed in this paper. It is clear from (4) and (5) that the results just stated are covered by Theorems 1 and 2 . It has been shown elsewhere that (7) is sufficient for the summability $(C, \beta), \beta>\alpha$, of the Fourier series whenever it is summable $(C)$. This result appears again (Theorem 3) as a corollary of Theorem 1, but for the more general class of Fourier series summable $(A)$. There are "converse" results in which boundedness $(C)$ of the sequence $n A_{n}$, or a more general condition, may be taken as hypothesis (Theorem 4), and the problem of the boundedness $(C)$ of $n A_{n}$ may also be solved in a "necessary and sufficient" form (Theorem 5). Finally this problem and the corresponding one for allied series may be regarded as the starting point of a sequence of more delicate problems of the same nature (Theorems 7 and 8).

2. Before proving the theorems of this section we state as lemmas some results which help to explain the hypotheses.*

LEмма 1. If $\alpha>-1$, necessary and sufficient conditions that (2) should hold and $\Phi_{\alpha+1}(+0)=0$ are that

$$
\frac{1}{t} \int_{0}^{t} u\left|d \phi_{\alpha+1}(u)\right|=O(1) \dagger
$$

and $\phi_{k}(t)=O(1)$ for some $k \geqq 0$.

\footnotetext{
* These have been given elsewhere, Bosanquet, 8.

$\dagger$ This integral is to be interpreted in the sense $\lim _{\epsilon \rightarrow 0} \int_{\epsilon}^{t}$.
} 
LEMMA 2. Necessary and sufficient conditions that (3) should hold are that (9) should hold with $\alpha=-1$ and $\phi_{k}(t)=O(1)$ for some $k \geqq 0$.

LEMMA 3. If $\alpha \geqq-1$, and (9) holds, then it still holds if $\alpha$ is replaced by $\beta>\alpha$.

There are analogous results with series in place of functions. We also require the following lemma.

LEMMA 4. If $\beta>\alpha>-1$, and (i) $\Phi_{\alpha+1}(t)$ is of bounded variation in an interval $(0, \eta)$, (ii) $\Phi_{\alpha+1}(+0)=0$, then $\Phi_{\beta}(t)$ exists for almost all $t$ in $(0, \eta)$, and satisfies the relation

$$
\Phi_{\beta}(t)=\frac{1}{\Gamma(\beta-\alpha)} \int_{0}^{t}(t-u)^{\beta-\alpha-1} d \Phi_{\alpha+1}(u) .
$$

TheOREM 1. If $\alpha \geqq 0$, and

$$
\frac{1}{t} \int_{0}^{t} u\left|d \phi_{\alpha}(u)\right|=O(1)^{*}
$$

in the interval $(0, \pi)$, then $n A_{n}=O(1)(C, \alpha+\delta)$, for every $\delta>0 . \dagger$

There is an analogous theorem with $o$ in place of $O$, and, more generally, we have the following result. $\ddagger$

THEOREM 1a. If $\alpha \geqq 0$, (11) holds in the interval $(0, \pi)$ and

$$
\frac{1}{t} \int_{0}^{t}\left|u d \phi_{\alpha}(u)+s d u\right|=o(1)
$$

as $t \rightarrow+0$, then $n A_{n} \rightarrow s(C, \alpha+\delta)$, for every $\delta>0$.

When $\alpha \geqq 1$ it is enough to consider the analogous theorem for Rieszian means. We therefore begin by proving the following theorem, which is the same in principle, but rather simpler in detail.

* It is easily seen, by integration by parts, that (11) is equivalent to $\int_{0}^{t} u^{\rho}\left|d \phi_{\alpha}(u)\right|=O\left(t^{\rho}\right), \rho>0$, or

$$
\int_{t}^{\pi} u^{-\rho}\left|d \phi_{\alpha}(u)\right|=O\left(t^{-\rho}\right), \rho>0 .
$$

$\dagger$ When $\alpha+\delta \geqq 1$ the conclusion is still true if (11) holds in some interval $(0, \eta)$.

$\ddagger$ If we observe that

$$
\log \left|\frac{1}{2} \operatorname{cosec} \frac{1}{2} t\right| \sim \sum_{1}^{\infty} \frac{\cos n t}{n}
$$

we easily see that Theorem 1a may be reduced to the $o$ analogue of Theorem 1 by replacing $\phi(t)$ by $\phi(t)-s \log \left|t^{-1}\right|$. 
THEOREM 2. If $\alpha \geqq 1$ and

$$
\frac{1}{t} \int_{0}^{t} u\left|\phi_{\alpha}^{\prime}(u)\right| d u=O(1)
$$

in an interval $(0, \eta)$, then

$$
\sum_{n<\omega} n A_{n}=O(\omega) \quad(C, \alpha-1+\delta)^{*}
$$

as $\omega \rightarrow \infty$, for every $\delta>0$.

Proof of Theorem 2. We have

$$
\begin{aligned}
\beta \omega^{-\beta} \sum_{n<\omega}(\omega-n)^{\beta-1} n A_{n} & =\beta \omega^{-\beta} \sum_{n<\omega}(\omega-n)^{\beta-1}\{\omega-(\omega-n)\} A_{n} \\
& =\beta\left\{r_{\beta-1}(\omega)-r_{\beta}(\omega)\right\} \\
& =\omega r_{\beta}^{\prime}(\omega),
\end{aligned}
$$

and so we have to show that $\omega r_{\beta}^{\prime}(\omega)=O(1)$ as $\omega \rightarrow \infty$, for $\beta>\alpha$. There is no loss of generality in supposing that $\beta \leqq \alpha+1$. Now it has been shown elsewhere that

where

$$
\frac{1}{2} \pi r_{\beta}(\omega)=\omega \int_{0}^{\infty} \phi_{\alpha}(t) J_{\beta}^{\alpha}(\omega t) d t=\int_{0}^{\infty} \phi_{\alpha}\left(\frac{u}{\omega}\right) J_{\beta}^{\alpha}(u) d u,
$$

$$
J_{\beta}^{\alpha}(x)=\frac{(-1)^{h+1} x^{h+1}}{\Gamma(1+h-\alpha) \Gamma(\alpha+1)} \int_{1}^{\infty}(t-1)^{h-\alpha} \gamma_{1+\beta}^{(h+1)}(x t) d t,
$$

$h$ being the greatest integer not greater than $\alpha$, and $\gamma_{1+\beta}(x)$ being given by

$$
\gamma_{1+\beta}(x)=\int_{0}^{1}(1-u)^{\beta} \cos x u d u . \ddagger
$$

It has also been shown $\S$ that, for $x>0$,

$$
\left|J_{\beta}^{\alpha}(x)\right|\left\{\begin{array}{l}
\leqq A \\
\leqq A x^{\alpha-\beta-1}
\end{array}\right.
$$

By differentiation under the integral sign we obtain\|

* This is equivalent to $n A_{n}=O(1)(C, \alpha+\delta)$. Cf. Hobson, 14, pp. 90-98.

$\dagger$ Bosanquet, 6 . The formula is valid for $\beta \geqq \alpha \geqq 0$.

23.

$\ddagger \gamma_{\alpha}(x)=\Gamma(\alpha) x^{-\alpha} C_{\alpha}(x)$, where $C_{\alpha}(x)$ is Young's generalization of the cosine function. Young,

$\S$ See Bosanquet, 3 and 6 . Here and elsewhere $A$ denotes some number independent of the variable, or variables, under consideration, and is not necessarily the same at each occurrence.

$\|$ It will be seen that the resulting integral converges uniformly for $\omega \geqq \epsilon>0$, when $\beta>\alpha$. 


$$
\frac{1}{2} \pi \omega r_{\beta}^{\prime}(\omega)=-\frac{1}{\omega} \int_{0}^{\infty} u \phi_{\alpha}^{\prime}\left(\frac{u}{\omega}\right) J_{\beta}^{\alpha}(u) d u=-\omega \int_{0}^{\infty} t \phi_{\alpha}^{\prime}(t) J_{\beta}^{g}(\omega t) d t .
$$

Now write, for $\omega>\pi^{-1}$,

$$
-\frac{1}{2} \pi \omega r_{\beta}^{\prime}(\omega)=\omega \int_{0}^{\infty}=\omega \int_{0}^{\omega^{-1}}+\omega \int_{\omega^{-1}}^{\pi}+\omega \int_{\pi}^{\infty}=I_{1}+I_{2}+I_{8} .
$$

Then we have, by (13) and (17),

$$
\left|I_{1}\right| \leqq A \omega \int_{0}^{\omega^{-1}}\left|t \phi_{\alpha}^{\prime}(t)\right| d t=O(1)
$$

and, integrating by parts in the usual manner,

$$
\left|I_{2}\right| \leqq A \omega^{\alpha-\beta} \int_{\omega^{-1}}^{\pi}\left|t \phi_{\alpha}^{\prime}(t)\right| t^{\alpha-\beta-1} d t=O(1)
$$

for $\beta>\alpha$. Finally, if we write

$$
I_{3}=\sum_{\nu=1}^{\infty} \omega \int_{\nu \pi}^{(\nu+1) \pi}=\sum_{\nu=1}^{\infty} I_{8}^{(\nu)},
$$

we can show from the periodicity of $\phi(t)$ that $I_{3}(\nu)=O\left(\omega^{\alpha-\beta} \nu^{\alpha-\beta-1}\right)$, uniformly in $\nu$, and hence $I_{3}=O\left(\omega^{\alpha-\beta}\right)$, for $\beta>\alpha$. This completes the proof.

Proof of Theorem 1. In what follows we need only consider the case $0<\alpha<\beta \leqq 1$. The case $\alpha=0$ follows from Lemma 3, with $\alpha$ in place of $\alpha+1$, but a direct proof proceeds along similar lines.

We first observe that $\Phi_{\alpha}(t)$ is of bounded variation in $(0, \pi)$, and $\Phi_{\alpha}(+0)$ $=0$. For, if $0<t<\pi$, we have by (11), writing $\Phi^{*}(t)=\int_{0}^{t} u\left|d \phi_{\alpha}(u)\right|$,

$$
\begin{aligned}
\int_{t}^{\pi}\left|d \phi_{\alpha}(u)\right| & =\int_{t}^{\pi} \frac{1}{u}\left|u d \phi_{\alpha}(u)\right| \\
& =\left[\frac{1}{u} \Phi^{*}(u)\right]_{t}^{\pi}+\int_{t}^{\pi} \Phi^{*}(u) \frac{d u}{u^{2}} \\
& =O(1)+O\left(\log \frac{\pi}{t}\right) .
\end{aligned}
$$

It follows that

$$
\phi_{\alpha}(t)=O\left(\log \frac{2 \pi}{t}\right)
$$

and hence $\Phi_{\alpha}(+0)=0$. Hence also, for $0<t<\dot{\pi}$, 


$$
\begin{aligned}
\Gamma(\alpha+1) \int_{t}^{\pi}\left|d \Phi_{\alpha}(u)\right| & =\int_{t}^{\pi}\left|d\left\{u^{\alpha} \phi_{\alpha}(u)\right\}\right| \\
& \leqq \int_{t}^{\pi} u^{\alpha}\left|d \phi_{\alpha}(u)\right|+\alpha \int_{t}^{\pi} u^{\alpha-1}\left|\phi_{\alpha}(u)\right| d u \\
& =O(1)+O\left(t^{\alpha} \log \frac{2 \pi}{t}\right) \\
& =O(1) .
\end{aligned}
$$

Now, if $\kappa^{\alpha}(n ; t)+i \bar{\kappa}^{\alpha}(n, t)$ denotes the Cesàro mean of order $\alpha$ of the sequence

$$
\frac{1}{\pi}+\frac{2}{\pi} \sum_{n=1}^{n} e^{i n t}
$$

the Cesàro mean of order $\beta$ of $(2 / \pi) \sin n t$ is $\beta(n+\beta)^{-1} \bar{\kappa}^{\beta-1}(n, t)$. Since

$$
n A_{n}=\frac{2}{\pi} \int_{0}^{\pi} \phi(t) \frac{d}{d t}(\sin n t) d t
$$

we now have, by Lemma 4, with 0 in place of $\beta$ and $\alpha$ in place of $\alpha+1$,

$$
\begin{aligned}
\tau_{n}^{\beta} & =\frac{\beta}{n+\beta} \int_{0}^{\pi} \phi(t) \frac{d}{d t} \bar{\kappa}^{\beta-1}(n, t) d t \\
& =O\left(\frac{1}{n}\right) \int_{0}^{\pi} \frac{d}{d t} \bar{\kappa}^{\beta-1}(n, t) d t \int_{0}^{t}(t-u)^{-\alpha} d \Phi_{\alpha}(u) \\
& =O\left(\frac{1}{n}\right) \int_{0}^{\pi} d \Phi_{\alpha}(u) \int_{u}^{\pi}(t-u)^{-\alpha} \frac{d}{d t} \bar{\kappa}^{\beta-1}(n, t) d t \\
& =O\left(\frac{1}{n}\right)\left[\Phi_{\alpha}(u) J(n, u)\right]_{0}^{\pi}+O\left(\frac{1}{n}\right) \int_{0}^{\pi} \Phi_{\alpha}(u) \frac{d}{d u} J(n, u) d u,
\end{aligned}
$$

where

$$
J(n, u)=\int_{u}^{\pi}(t-u)^{-\alpha} \frac{d}{d t} \bar{\kappa}^{\beta-1}(n, t) d t,
$$

provided the last two steps be justified.

We next show that, for $0<u<\pi$,

$$
|J(n, u)|\left\{\begin{array}{l}
\leqq A n^{1+\alpha} \\
\leqq A n^{1+\alpha-\beta} u^{-\beta} .
\end{array}\right.
$$

Now, for $0<t<\pi, 0<\beta \leqq 1$, we have*

* Cf. Zygmund, 27, and Gergen, 10. Both these papers are concerned with $\kappa^{\beta-1}(n, t)$, but contain enough analysis to show how (21) and (22) are obtained. 


$$
\left|\bar{\kappa}^{\beta-1}(n, t)\right|\left\{\begin{array}{l}
\leqq A n \\
\leqq A n^{1-\beta} t^{-\beta}
\end{array}\right.
$$

and

$$
\left|\frac{d}{d t}{ }^{-\beta-1}(n, t)\right|\left\{\begin{array}{l}
\leqq A n^{2} \\
\leqq A n^{2-\beta} t^{-\beta}
\end{array}\right.
$$

Hence writing, for $u+n^{-1}<\pi$, $^{*}$

$$
J(n, u)=\int_{u}^{u+n^{-1}}+\int_{u+n^{-1}}^{\pi}=J_{1}+J_{2}
$$

we have, by (22),

$$
\begin{aligned}
\left|J_{1}\right| & \leqq A n^{2-\beta} \int_{u}^{u+n^{-1}}(t-u)^{-\alpha} \min \left(n^{\beta}, t^{-\beta}\right) d t \\
& \leqq A n^{2-\beta} \min \left(n^{\beta}, u^{-\beta}\right) \int_{u}^{u+n^{-1}}(t-u)^{-\alpha} d t \\
& =O\left(n^{1+\alpha-\beta}\right) \min \left(n^{\beta}, u^{-\beta}\right),
\end{aligned}
$$

and, by the second mean-value theorem,

$$
\begin{aligned}
J_{2} & =\left(\frac{1}{n}\right)^{-\alpha} \int_{u+n^{-1}}^{\zeta} \frac{d}{d t} \bar{\kappa}^{\beta-1}(n, t) d t \dagger \\
& =n^{\alpha} O\left(n^{1-\beta}\right) \min \left(n^{\beta}, u^{-\beta}\right)
\end{aligned}
$$

by (21). This establishes (20).

Returning now to the main theme of the proof, we see that the inversion of the repeated integral is justified, the resulting integral being absolutely convergent, $\ddagger$ and we obtain

* When $u+n^{-1} \geqq \pi$ the second integral does not occur, and the argument is simpler.

$\dagger$ Where $u+n^{-1}<\zeta<\pi$.

$\ddagger$ Since, by (22),

$$
\begin{gathered}
\int_{0}^{\pi}\left|d \Phi_{\alpha}(u)\right| \int_{u}^{\pi}(t-u)^{-\alpha}\left|\frac{d}{d t} \bar{\kappa}^{\beta-1}(n, t)\right| d t \\
\leqq A n^{2} \int_{0}^{\pi}\left|d \Phi_{\alpha}(u)\right| \int_{u}^{\pi}(t-u)^{-\alpha} d t \\
\leqq A n^{2} \int_{0}^{\pi}\left|d \Phi_{\alpha}(u)\right|
\end{gathered}
$$

for $\alpha<1$. We use the analogue for Stieltjes integrals of Fubini's theorem; see Bosanquet, 8. 


$$
\begin{aligned}
\tau_{n}^{\beta}=O\left(n^{\alpha-\beta}\right) & +O\left(\frac{1}{n}\right) \int_{0}^{\pi} \phi_{\alpha}(u) u^{\alpha} \frac{d}{d u} J(n, u) d u \\
=O\left(n^{\alpha-\beta}\right) & +O\left(\frac{1}{n}\right)\left[\phi_{\alpha}(u) \int_{0}^{u} v^{\alpha} \frac{d}{d v} J(n, v) d v\right]_{0}^{\pi} \\
& +O\left(\frac{1}{n}\right) \int_{0}^{\pi} d \phi_{\alpha}(u) \int_{0}^{u} v^{\alpha} \frac{d}{d v} J(n, v) d v
\end{aligned}
$$

It will be enough now to show that, for $0<u \leqq \pi$,

$$
\left|\int_{0}^{u} v^{\alpha} \frac{d}{d v} J(n, v) d v\right|\left\{\begin{array}{l}
\leqq A n^{1+\alpha} u^{\alpha} \\
\leqq A n^{1+\alpha-\beta} u^{\alpha-\beta}
\end{array}\right.
$$

For, if this be established, it will follow that the integrated term is

$$
O\left(\frac{1}{n}\right) \phi_{\alpha}(\pi) \int_{0}^{\pi} v^{\alpha} \frac{d}{d v} J(n, v) d v=O\left(n^{\alpha-\beta}\right),
$$

and, writing

$$
\frac{1}{n} \int_{0}^{\pi} d \phi_{\alpha}(u) \int_{0}^{u} v^{\alpha} \frac{d}{d v} J(n, v) d v=\frac{1}{n} \int_{0}^{n^{-1}}+\frac{1}{n} \int_{n^{-1}}^{\pi}=K_{1}+K_{2},
$$

we shall have, from (11), integrating by parts in the usual way,

$$
\left|K_{1}\right| \leqq A n^{\alpha} \int_{0}^{n^{-1}} u^{\alpha-1}\left|u d \phi_{\alpha}(u)\right|=O(1)
$$

and

$$
\left|K_{2}\right| \leqq A n^{\alpha-\beta} \int_{n^{-1}}^{\pi} u^{\alpha-\beta-1}\left|u d \phi_{\alpha}(u)\right|=O(1)
$$

for $\beta>\alpha$. The theorem will therefore be proved.

To establish (23), we first observe that

$$
\int_{0}^{u} v^{\alpha} \frac{d}{d v} J(n, v) d v=u^{\alpha} J(n, u)-\alpha \int_{0}^{u} v^{\alpha-1} J(n, v) d v
$$

and the first inequality follows from the first inequality (20). We next observe that, if $\phi(t) \equiv 1$, then $\phi_{\alpha}(t)=1$ for all $t$ and $\tau_{n}{ }^{\beta}=0$ for all $n$. Hence, following through our previous reasoning with this special value of $\phi(t)$, we find that

$$
0=O\left(n^{\alpha-\beta}\right)+\frac{\beta}{n+\beta} \int_{0}^{\pi} v^{\alpha} \frac{d}{d v} J(n, v) d v
$$

If we now write 


$$
\int_{0}^{u} v^{\alpha} \frac{d}{d v} J(n, v)=\int_{0}^{\pi}-\int_{u}^{\pi}=J^{\prime}-J^{\prime \prime},
$$

we have $J^{\prime}=O\left(n^{1+\alpha-\beta}\right)$, and finally, observing that

$$
\int_{u}^{\pi} v^{\alpha} \frac{d}{d v} J(n, v) d v=\left[v^{\alpha} J(n, v)\right]_{u}^{\pi}-\alpha \int_{u}^{\pi} v^{\alpha-1} J(n, v) d v,
$$

we obtain, from the second inequality (20), $J^{\prime \prime}=O\left(n^{1+\alpha-\beta}\right)+O\left(n^{1+\alpha-\beta} u^{-\beta}\right)$. The second inequality (23) now follows, for $0<u \leqq \pi$.

This completes the proof.

We have as a corollary of Theorem 1, after our remarks in the introduction, the following theorem.

THEOREM 3. If $\alpha \geqq 0$ and (11) holds in the interval $(0, \pi)$, then the Fourier series of $f(t)$ is summable $(C, \alpha-1+\delta)$ at the point $t=x$, for every $\delta>0$, if it is summable $(A)$.

3. The next theorem shows that a condition of the type (11) is also necessary for the boundedness $(C)$ of the sequence.

We confine ourselves to the Rieszian form of the theorem.

Theorem 4. If $\alpha \geqq 0$ and

$$
\frac{1}{\omega} \int_{0}^{\omega} u\left|d r_{\alpha}(u)\right|=O(1)^{*}
$$

as $\omega \rightarrow \infty$, then $\phi_{\beta}^{\prime}(t)=O(1)$ in the interval $(0, \pi)$, for $\beta>\alpha+1$.

There is an analogous result with $o$ in place of $O$.

It follows from (24) that $r_{\alpha}(\omega)=O(\log \omega)$ as $\omega \rightarrow \infty$. If then $\beta>\alpha+1$, $t>0$, and $h$ is the greatest integer not greater than $\alpha$, we have, by an argument used elsewhere, $†$ since $A_{n}=o(1)$,

* The condition is satisfied in particular if $\alpha \geqq 1$ and $\omega r_{\alpha}^{\prime}(\omega)=O(1)$, i.e.,

$$
\sum_{n<\omega} n A_{n}=O(\omega)(C, \alpha-1),
$$

or, what is equivalent, $n A_{n}=O(1)(C, \alpha)$. When $\alpha=0$ (24) becomes

$$
\sum_{n<\omega} n\left|A_{n}\right|=O(\omega) \text {. }
$$

$\dagger$ See Bosanquet, 3 and 4. The argument was previously used with the hypothesis $R_{\alpha}(\omega)=o\left(\omega^{\alpha}\right)$. In the present case we have

as $\omega \rightarrow \infty$. Also

$$
\begin{aligned}
R_{\rho}(\omega) & =o\left(\omega^{\rho+1}\right), \quad 0 \leqq \rho \leqq \alpha-1, \\
& =O\left(\omega^{\alpha} \log \omega\right), \quad \alpha-1<\rho \leqq \alpha, \\
& =O\left(\omega^{\rho} \log \omega\right), \quad \rho \geqq \alpha, \\
& \gamma_{\beta}^{(\rho)}(x)=O\left(x^{-\rho-2}\right)+O\left(x^{-\beta}\right)
\end{aligned}
$$

as $x \rightarrow \infty$, and $\gamma_{\beta}{ }^{(\rho)}(x)=O(1)$ for $x>0$. The inversion of the repeated integral remains valid in the present case. 


$$
\begin{aligned}
\beta^{-1} \phi_{\beta}(t)= & -\int_{0}^{\infty} \gamma_{\beta}(t u) R_{0}(u) d u \\
= & {\left[\sum_{\rho=1}^{h+1} \frac{(-1)^{\rho}}{\Gamma(\rho+1)} t^{\rho} \gamma_{\beta}^{(\rho)}(t u) R_{\rho}(u)\right]_{0}^{\infty} } \\
& \quad+\frac{(-1)^{h+2} t^{h+2}}{\Gamma(h+2)} \int_{0}^{\infty} \gamma_{\beta}^{(h+2)}(t u) R_{h+1}(u) d u \\
= & \frac{(-1)^{h+2} t^{h+2}}{\Gamma(1+h-\alpha) \Gamma(\alpha+1)} \int_{0}^{\infty} \gamma_{\beta}^{(h+2)}(t u) d u \int_{0}^{u}(u-v)^{h-\alpha} R_{\alpha}(v) d v \\
= & \frac{(-1)^{h+2} t^{h+2}}{\Gamma(1+h-\alpha) \Gamma(\alpha+1)} \int_{0}^{\infty} R_{\alpha}(v) d v \int_{v}^{\infty}(u-v)^{h-\alpha} \gamma_{\beta}^{(h+2)}(t u) d u \\
= & t \int_{0}^{\infty} r_{\alpha}(v) I_{\beta}^{\alpha}(t v) d v=\int_{0}^{\infty} r_{\alpha}^{\prime}\left(\frac{w}{t}\right) I_{\beta}^{\alpha}(w) d w,
\end{aligned}
$$

where

$$
I_{\beta}^{\alpha}(x)=\frac{(-1)^{h+2} x^{h+1}}{\Gamma(1+h-\alpha) \Gamma(\alpha+1)} \int_{1}^{\infty}(y-1)^{h-\alpha} \gamma_{\beta}^{(h+2)}(x y) d y .
$$

It has also been shown* that, for $x>0, \beta>\alpha+1 \geqq 1$,

$$
\left|I_{\beta}^{\alpha}(x)\right|\left\{\begin{array}{l}
\leqq A \\
\leqq A x^{\alpha-\beta}
\end{array}\right.
$$

It follows, by differentiation under the integral sign, $\nmid$ that

$$
\beta^{-1} t \phi_{\beta}^{\prime}(t)=-\frac{1}{t} \int_{0}^{\infty} I_{\beta}^{\alpha}(w) w d r_{\alpha}\left(\frac{w}{t}\right)=-t \int_{0}^{\infty} I_{\beta}^{\alpha}(t v) v d r_{\alpha}(v) .
$$

Therefore, writing

we have

$$
-\beta^{-1} t \phi_{\beta}^{\prime}(t)=t \int_{0}^{t^{-1}}+t \int_{t^{-1}}^{\infty}=L_{1}+L_{2},
$$

$$
\left|L_{1}\right| \leqq A t \int_{0}^{t^{-1}}\left|v d v_{a}(v)\right|=O(1)
$$

and

$$
\left|L_{2}\right| \leqq A t^{1+\alpha-\beta} \int_{t-1}^{\infty} v^{\alpha-\beta}\left|v d v_{\alpha}(v)\right|=O(1)
$$

for $\beta>\alpha+1$. This proves the theorem.

- Bosanquet, 3.

$\dagger$ The resulting integral converges uniformly for $t \geqq \epsilon>0$, when $\beta>\alpha+1$. 
Combining Theorems 1 and 4 together we now have the following theorem.

THEOREM 5. A necessary and sufficient condition that $n A_{n}=O(1)(C)$ is that, for some $\kappa \geqq 0, \phi_{k}(t)$ should be absolutely continuous except at $t=0$ and $t \phi_{k}^{\prime}(t)=O(1)$ in the interval $(0, \pi)$.

There is an analogous result with $o$ in place of $O$.

We also add the following theorem.

THEOREM 6. If $\alpha \geqq 0$ and

$$
\frac{1}{\omega} \int_{0}^{\omega}\left|r_{\alpha}(u)\right|=O(1)
$$

as $\omega \rightarrow \infty$, then $\phi_{\beta}(t)=O(1)$ in the interval $(0, \pi)$, for $\beta>\alpha+1$.

There is an analogous result with $o$ in place of $O .^{*}$

It follows from (26), by the analogue of Lemma 1, that (24) is satisfied, with $\alpha+1$ in place of $\alpha$, and $\sum A_{n}$ is bounded $(C)$. Hence $t \phi_{\beta+1}^{\prime}(t)=O(1)$ for $\beta>\alpha+1$, by Theorem 4 , and $\phi(t)=O(1)(C)$. $\dagger$ The result may now be obtained from (5).

4. Finally we can generalize the Rieszian form of Theorem 5 as follows.

THEOREM 7. If $\lambda$ is a non-negative integer, a necessary and sufficient condition that

$$
\left(\omega \frac{d}{d \omega}\right)^{\lambda} r_{\beta}(\omega)=O(1) \text { as } \omega \rightarrow \infty,
$$

for some $\beta \geqq \lambda$, is that

$$
\left(t \frac{d}{d t}\right)^{\lambda} \phi_{k}(t)=O(1) \text { in the interval }(0, \pi)
$$

for some $\kappa \geqq \lambda$, where $\phi_{k}(t)$ is a $\lambda$ th integral except at $t=0$.

Suppose that for some $\alpha \geqq \lambda$ we have

$$
\left(t \frac{d}{d t}\right)^{\lambda} \phi_{\alpha}(t)=O(1) \text { for } 0<t<\pi .
$$

Then if $0 \leqq \mu \leqq \lambda$, we have

* For the case $\alpha=0$ see Bosanquet, 4. The hypothesis is satisfied in particular when $\sum A_{n}$ is summable $(C, \alpha)$. See Hardy and Littlewood, 11, Paley, 18, Verblunsky, 19, Wiener, 21, 22, Bosanquet, 3.

† Hardy and Littlewood, 11. 


$$
\left(t \frac{d}{d t}\right)^{\lambda-\mu} \phi_{\alpha}(t)=O\left\{\left(\log \frac{1}{t}\right)^{\mu}\right\} \text { as } t \rightarrow 0,
$$

and it is easily shown by induction that

$$
\frac{1}{2} \pi\left(\omega \frac{d}{d \omega}\right)^{\lambda} r_{\beta}(\omega)=(-1)^{\lambda} \omega \int_{0}^{\infty}\left(t \frac{d}{d t}\right)^{\lambda} \phi_{\alpha}(t) J_{\beta}^{\alpha}(\omega t) d t
$$

for $\beta>\alpha$. If, on the other hand, for some $\alpha \geqq \lambda$ we have

then

$$
\left(\omega \frac{d}{d \omega}\right)^{\lambda} r_{\alpha}(\omega)=O(1) \text { as } \omega \rightarrow \infty,
$$

$$
\left(\omega \frac{d}{d \omega}\right)^{\lambda-\mu} r_{\alpha}(\omega)=O\left\{(\log \omega)^{r}\right\} \text { as } \omega \rightarrow \infty,
$$

and we obtain, for $\beta>\alpha+1$,

$$
\beta^{-1}\left(t \frac{d}{d t}\right)^{\lambda} \phi_{\beta}(t)=(-1)^{\lambda} t \int_{0}^{\infty}\left(u \frac{d}{d u}\right)^{\lambda} r_{\alpha}(u) I_{\beta}^{\alpha}(t u) d u .
$$

The result follows from (27) and (28) by arguments analogous to those of Theorems 2 and 4.

The analogue of Theorem 7 for conjugate series is as follows.*

THEOREM 8. If $\lambda$ is a non-negative integer, a necessary and sufficient condition that

$$
\left(\omega \frac{d}{d \omega}\right)^{\lambda} \bar{r}_{\beta}(\omega)=O(1) \text { as } \omega \rightarrow \infty,
$$

for some $\beta \geqq \lambda$, is that

$$
\left(t \frac{d}{d t}\right)^{\lambda} \theta_{k}(t)=O(1) \text { in the interval }(0, \pi)
$$

for some $k \geqq \lambda$, where $\theta_{k}(t)$ is a $\lambda$ th integral except at $t=0$.

It is interesting to note that, by (6), if $k \geqq 1, \dagger$

$$
t \frac{d}{d t} \theta_{k}(t)=-\frac{2}{\pi} \psi_{k}(t)
$$

and so, when $\lambda \geqq 1$, the condition in Theorem 8 takes the form

* The previously known cases were $\lambda=0$ or 1 . Hardy and Littlewood, 12.

$\dagger$ When $0 \leqq k<1$ this is true except possibly in a set of measure zero. 


$$
\left(t \frac{d}{d t}\right)^{\lambda-1} \psi_{k}(t)=O(1)
$$

\section{REFERENCES}

1. A. F. Andersen, Studier over Cesdro's Summabilitetsmetode. Copenhagen, 1921.

2. L. S. Bosanquet, On Abel's integral equation and fractional integrals. Proceedings of the London Mathematical Society, (2), vol. 31 (1930), pp. 135-143.

3. - On the summability of Fourier series. Proceedings of the London Mathematical Society, (2), vol. 31 (1930), pp. 144-164.

4. - On strongly summable Fourier series. Journal of the London Mathematical Society, vol. 7 (1932), pp. 47-52.

5. - Note on the limit of a function at a point. Journal of the London Mathematical Society, vol. 7 (1932), pp. 100-105.

6. - On the Cesdro summation of Fourier series and allied series. Proceedings of the London Mathematical Society, (2), vol. 37 (1934), pp. 17-32.

7. - The absolute summability $(A)$ of Fourier series. Proceedings of the Edinburgh Mathematical Society, (2), vol. 4 (1934), pp. 12-17.

8. - - Some extensions of Young's criterion for the convergence of a Fourier series. Quarterly Journal of Mathematics (Oxford series), vol. 6 (1935), pp. 113-123.

9. L. S. Bosanquet and A. C. Offord, A local property of Fourier series. Proceedings of the London Mathematical Society, (2), vol. 40 (1935), pp. 273-280.

10. J. J. Gergen, Convergence and summability criteria for Fourier series. Quarterly Journal of Mathematics (Oxford series), vol. 1 (1930), pp. 252-275.

11. G. H. Hardy and J. E. Littlewood, Solution of the Cesdro summability problem for power series and Fourier series. Mathematische Zeitschrift, vol. 19 (1924), pp. 67-96.

12. - The allied series of a Fourier series. Proceedings of the London Mathematical Society, (2), vol. 24 (1925), pp. 211-246.

13. - Notes on the theory of series (VII): On Young's convergence criterion for Fourier series. Proceedings of the London Mathematical Society, (2), vol. 28 (1928), pp. 301-311.

13a. - Notes on the theory of series (XVI): Two Tauberian theorems. Journal of the London Mathematical Society, vol. 6 (1931), pp. 281-286.

14. E. W. Hobson, The Theory of Functions of a Real Variable. 2d edition, vol. 2, 1926.

15. E. Kogbetliantz, Sur les séries absolument sommables par la méthode des moyennes arithmétiques. Bulletin des Sciences Mathématiques, (2), vol. 49 (1925), pp. 234-256.

16. - Sommation des Séries et Intégrales Divergentes par les Moyennes Arithmétiques et Typiques. Mémorial des Sciences Mathématiques, vol. 51 (1931).

17. J. E. Littlewood, The converse of Abel's theorem on power series. Proceedings of the London Mathematical Society, (2), vol. 9 (1910), pp. 434-448.

18. R. E. A. C. Paley, On the Cesdro summability of Fourier series and allied series. Proceedings of the Cambridge Philosophical Society, vol. 26 (1930), pp. 173-203.

19. S. Verblunsky, Note on the sum of oscillating series. Proceedings of the Cambridge Philosophical Society, vol. 26 (1930), pp. 152-157.

20. - On the limit of a function at a point. Proceedings of the London Mathematical Society, (2), vol. 32 (1931), pp. 163-199.

21. N. Wiener, A type of Tauberian theorem applying to Fourier series. Proceedings of the London Mathematical Society, (2), vol. 30 (1929), pp. 1-8.

22. - Tauberian theorems. Annals of Mathematics, (2), vol. 33 (1932), pp. 1-100.

23. W. H. Young, On infinite integrals involving a generalization of the sine and cosine functions. Quarterly Journal of Mathematics, vol. 43 (1912), pp. 161-177. 
24. - On the order of magnitude of the coefficients of a Fourier series. Proceedings of the Royal Society, (A), vol. 93 (1916), pp. 42-55.

25. - On the mode of approach to zero of the coefficients of a Fourier series. Proceedings of the Royal Society, (A), vol. 93 (1916), pp. 655-667.

26. - On the convergence of the derived series of a Fourier series. Proceedings of the London Mathematical Society, (2), vol. 17 (1918), pp. 195-236.

27. A. Zygmund, Sur un théorème de M. Gronwall. Bulletin de l'Académie Polonaise (Cracovie), (A), 1925, pp. 207-217.

\section{UNTYERSTTY COLLEge,}

LONDON, ENGLAND 